\title{
Case Report of Metastatic Head and Neck Hidradenoma
}

Alvin deTorres ${ }^{1}$, Bari Hoffman-Ruddy ${ }^{1}$, Erin Pearson Silverman ${ }^{2,4,6 *}$, Henry Ho ${ }^{3}$ and Christine Sapienza ${ }^{4,5}$

${ }^{1}$ University of Central Florida, USA

${ }^{2}$ University of Florida, USA

${ }^{3}$ The Florida Hospital Cancer Institute, Head and Neck Program, USA

${ }^{4}$ Jacksonville University, USA

${ }^{5}$ Brooks Rehabilitation Hospital, USA

${ }^{6}$ Malcom Randall Veterans Affairs, BRRC, USA

\begin{abstract}
Hidradenocarcinoma is a rare malignancy of the eccrine sweat glands. Existing case studies on this cancer focus on margin free dissection and, less frequently radiation therapy. We report on a unique case of a 43 year old female who had been previously treated via surgical and radiation therapy for hid adenocarcinoma of the right index finger, referred to our practice from radiation oncology, with complaints of a new left neck mass. Subsequent imaging studies confirmed the presence of a left sided node as well as numerous metastatic nodules present throughout the body. Chemotherapy was the recommended treatment. We review the limited literature regarding surgical versus nonsurgical approaches for early detection and treatment of hid adenocarcinomas. The patient's refusal of recommended treatment highlights the aggressive nature of this rare cancer.
\end{abstract}

Keywords: Hidradenocarcinoma; Surgery; Metastasis; Head and Neck

\section{Introduction}

Hidradenocarcinoma is a rare malignancy of the eccrine sweat glands occurring in only one in every 13,000 dermatopathology specimens [1]. They most frequently occur on the head and neck and less commonly in the limbs. The mean age of onset is 50 years and there is no apparent predilection of sex [1].

The natural history of hidradenocarcinoma disease has been variably described from indolent to aggressive [2] with a mean 5 year survival rate from time of diagnosis of $73.9 \%$. Local recurrence is common and the disease will often metastasize to regional lymph nodes by the time of diagnosis, spreading to distant tissues in approximately $60 \%$ of patients and ultimately resulting in death in $40 \%$ of those diagnosed [2].

We present a unique case of a woman with a history of hidradenocarcinoma of the right index finger, confirmed via biopsy, who presented to our otolaryngology clinic for evaluation of a neck mass. This case report highlights the rarity of this malignant carcinoma, illustrates the aggressive course of the disease from regional nodal involvement to diffuse, widespread metastatic disease, emphasizing the importance of margin-free resection, as well as the need to evaluate regional nodes for detection of early subclinical metastases.

\section{Case Report}

The patient is a 43-year old female with a history of hidradenocarcinoma of the right medial index finger who was referred from radiation oncology to our otolaryngology clinic for evaluation of a new neck mass. Nine months prior to presentation, she underwent excision of a right index finger lesion by a hand surgeon. This lesion was later found to be a hidradenocarcinoma by histopathologic evaluation. Tumor involvement of the margins was found and wide local excision of the previous site was recommended. However, the patient declined surgery due to cosmetic and functional concerns and instead opted for ten weeks of adjuvant radiotherapy six weeks after her original surgery. Her radiation course was complicated by multiple interruptions secondary to severe blistering.

Five and a half months after her initial surgery, lymphadenopathy of the right axilla occurred which was positive for metastatic hidradenocarcinoma, confirmed via needle biopsy. Positive Emission Tomography (PET) scan from the skull to thigh revealed isolated involvement of the right axilla but revealed no other sites of abnormal hypermetabolism (Figure 1). Lymph node dissection of the right axilla was performed with subsequent diagnosis of hidradenocarcinoma within multiple nodes. Histopathology examination of the specimen found no evidence of extranodal soft tissue extension. Following surgery, she received external beam radiation to the right axilla and right supraclavicular fossa for a total of 50.4 Gy. Two weeks after

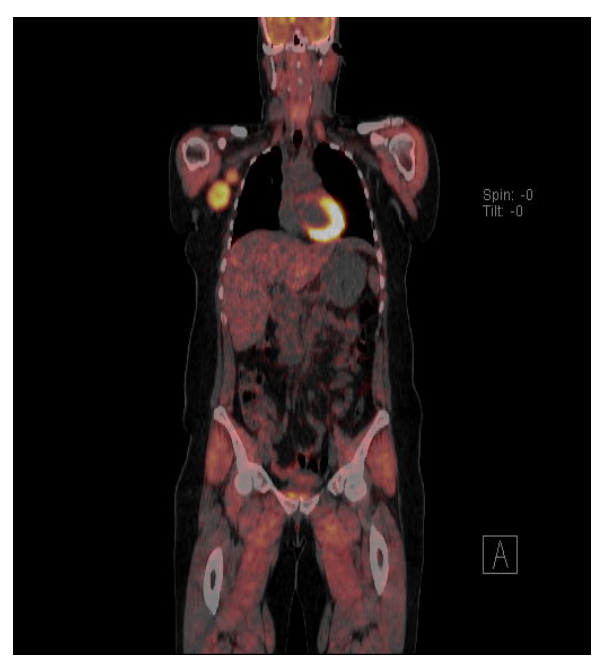

Figure 1: PET scan revealing axial involvement.

*Corresponding author: Erin Pearson Silverman, University of Central Florida PO Box 100144, Gainesville, FL, 32610, USA, Tel: 352-339-4100; E-mail epearson@ufl.edu

Received October 22, 2014; Accepted November 14, 2014; Published November 16,2014

Citation: Torres A, Hoffman-Ruddy B, Silverman EP, Ho H, Sapienza C (2014) Case Report of Metastatic Head and Neck Hidradenoma. J Clin Case Rep 4: 446. doi:10.4172/2165-7920.1000446

Copyright: ( $) 2014$ Torres A, et al. This is an open-access article distributed under the terms of the Creative Commons Attribution License, which permits unrestricted use, distribution, and reproduction in any medium, provided the original author and source are credited. 


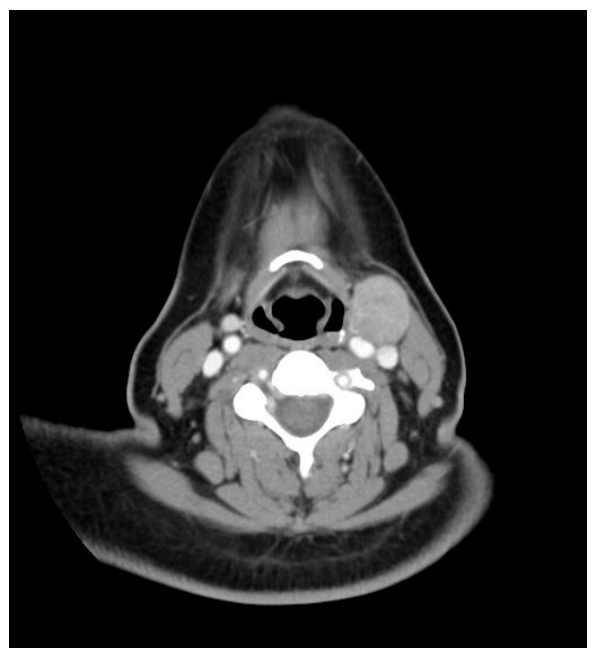

Figure 2: Neck CT revealing genioglossal involvement.

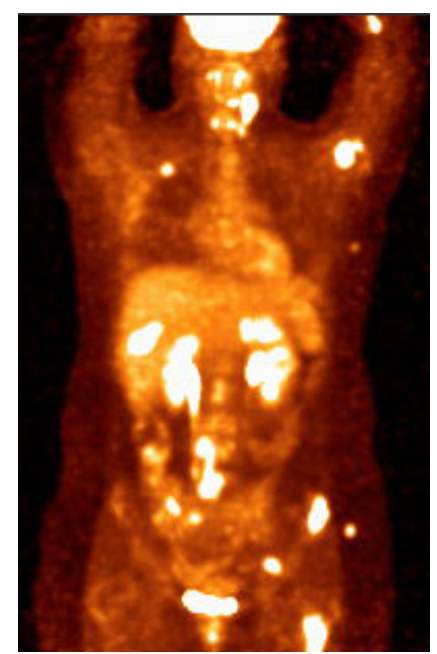

Figure 3: Systemic hype.

completion, the patient noticed a mass in the contralateral neck and was referred to our practice for otolaryngology evaluation.

At the time she presented for otolaryngologic evaluation, the patient had no complaints other than her left neck mass. Physical exam revealed a $3 \mathrm{~cm}$, mobile, round level II node without involvement of the overlying skin in the left neck. Other than the expected post-treatment changes in the right axilla and index finger, she was otherwise wellappearing. To evaluate her for distant disease and surgical candidacy for neck dissection, Computerized Tomography (CT) scan of the head and neck and PET scan of the skull to thigh were performed. Neck CT revealed a $1.1 \mathrm{~cm}$ lesion of the left floor of mouth with infiltration of the left genioglossus muscle, a $3.4 \mathrm{~cm}$ left level III node, a $0.8 \mathrm{~cm}$ left level II node, and a $1.0 \mathrm{~cm}$ left level IV node (Figure 2). The PET scan revealed numerous hypermetabolic nodules throughout the body including nodes in the head and neck coincident with the $\mathrm{CT}$ and in the bilateral axilla (Figure 3). Fine-Needle Aspiration (FNA) of the left neck nodes was consistent with metastatic hidradenocarcinoma. In light of these findings of wide spread metastatic disease, the decision was made to forego neck dissection and proceed with systemic chemotherapy.

\section{Discussion}

Sweat glands are classified as apocrine or eccrine glands based on location and histology. Eccrine glands are found in the palms and soles, head, trunk, and extremities while apocrine glands are found in the axilla, anogenital area, mammary tissues, and eyelids [3]. Malignant carcinomas of the eccrine sweat glands occur in 1 of every 13,000 dermatopathology specimens of which hidradenocarcinoma is one histological type [1].

Margin-free resection, sentinel lymph node biopsy, and long-term follow are essential to successful treatment of hidradenocarcinoma [4]. Complete margin-free excision can be accomplished through several modalities. Successful margin-free resection through simple and wide-local excision of hidradenocarcinoma of the finger has been described [4]. On the nose and scalp, Mohs micrographic surgery has been effectively used $[2,5]$. In the present case, tumor involvement of the margin was found and wide local excision of the previous site was offered by a plastic surgeon but was declined by the patient. In place of surgery, the patient opted to pursue radiation.

Data on the use of radiation in treating hidradenocarcinoma, especially in light of margin involvement, is limited and conflicting. Harari et al. [6] describe two cases of hidradenocarcinoma treated with surgical excision with positive margin involvement followed with radiation to the tumor bed and regional nodes and found 27 and 35 months of disease-free survival. In a large retrospective analysis of 1045 patients with eccrine gland carcinoma, other investigations have found no significant difference in 5-year survival when adjuvant radiotherapy was used.

As previously mentioned, hidradenocarcinoma primarily metastasizes via regional lymphatics at a rate as high as $50 \%$ [7]. In the presence of regional lymph node involvement or distant metastasis, survival is limited to 5 to 24 months even with wide excision [4]. The absence of nodal disease indicates better prognosis.

Unless there was clinically evident lymph node involvement, regional lymph nodes have not been evaluated surgically or histologically on a consistent basis in the literature. A large retrospective study including 236 cases of hidradenocarcinoma, 0 nodes were positive in $10.2 \%$ of cases, 1 or more were positive in $1.8 \%$, while the majority of hidradenocarcinoma cases had unknown nodal status (88\%) [8]. One limitation mentioned by the authors is that the majority of cases included in the review predated the time of routine sentinel lymph node biopsy. Some reports, including the present case, did not initially evaluate the regional nodes further because no clinical lymphadenopathy was found on exam and the pathologic findings from the initial surgery were low grade [9]. Others advocate elective lymph node dissection or radiation to clinically uninvolved regional nodes due to the high rate of metastasis [10]. An alternative is sentinel lymph node mapping and biopsy which has been shown to detect subclinical metastatic disease. This may increase clinical staging accuracy, allow early treatment, and avoid the morbidity of a formal dissection if further surgery is not needed [8]. However, the prognostic and therapeutic impact of sentinel lymph node biopsy has not been formally studied for sweat gland carcinomas and remains to be uncertain.

Hid adenocarcinoma is a rare and aggressive malignant neoplasm of the eccrine sweat glands with high rates of local recurrence and metastasis. The primary treatment consists of complete surgical excision with full evaluation of the margin. To date, nonsurgical treatment modalities have not been consistently shown to prevent 
Citation: Torres A, Hoffman-Ruddy B, Silverman EP, Ho H, Sapienza C (2014) Case Report of Metastatic Head and Neck Hidradenoma. J Clin Case Rep 4: 446. doi:10.4172/2165-7920.1000446

recurrence or metastasis when local tumor at the margin is found. However, given the aggressive nature of this tumor, and the tendency toward nodal metastasis, early detection of subclinical disease with sentinel node biopsy, leading to directed node dissection or adjuvant radiation therapy, may lead to improved outcomes for these patients. Whereas early case reports of hid adenocarcinoma detailed advanced metastatic disease due to delayed or unknown diagnosis, the presented case presents a clear clinical history from initial detection, through treatment, and into ultimate exacerbation with multiple noted metastasis. In this atypical case, where surgical standard of care treatment was refused by the patient, the aggressive nature of this cancer was demonstrated with widespread metastasis, evidence of which has been rarely documented in previous literature.

\section{References}

1. Mehregan AH, Hashimoto K, Rahbari H (1983) Eccrine adenocarcinoma. A clinicopathologic study of 35 cases. Arch Dermatol 119: 104-114.

2. Yave R, Hinshaw M, Rao V, Hartig GK, Harari PM (2009) Hidradenomas and a hidradenocarcinoma of the scalp managed using MohsmicrographicMohs micrographic surgery and a multidisciplinary approach: case reports and review of the literature. Dermatol Surg. 35: 273-281.
3. Matloub HS, Cunningham MW, Yousif NJ, Sanger JR, Romano JA, et al (1988) Eccrine porocarcinoma. Ann Plast Surg 20: 351-355.

4. Nazerali RS, Tan C, Fung MA, Chen SL, Wong MS (2013) Hidradenocarcinoma of the Finger. Ann Plast Surg .

5. Rubenzik M, Keller M, Humphreys T (2010) Mohs micrographic surgery for hidradenocarcinoma on a rhinophymatous nose: a histologic conundrum. Dermatol Surg 36: 2075-2078.

6. Harari PM, Shimm DS, Bangert JL, Cassady JR (1990) The role of radiotherapy in the treatment of malignant sweat gland neoplasms. Cancer 65: 1737-1740.

7. Delgado R, Kraus D, Coit DG, Busam KJ (2003) Sentinel lymph node analysis in patients with sweat gland carcinoma. Cancer 97: 2279-2284.

8. Avraham JB, Villines D, Maker VK, August C, Maker AV (2013) Survival after resection of cutaneous adnexal carcinomas with eccrine differentiation: risk factors and trends in outcomes. J Surg Oncol 108: 57-62.

9. Shu K, Xiao Q, Büchele F, Zhang S, Jiang W, et al. (2012) Diagnosis and treatment of clear cell hidradenocarcinoma of the scalp. J Huazhong Univ Sci Technolog Med Sci 32: 931-936.

10. Ohta M, Hiramoto M, Fujii M, Togo T (2004) Nodular hidradenocarcinoma on the scalp of a young woman: case report and review of literature. Dermatol Surg 30: 1265-1268. 